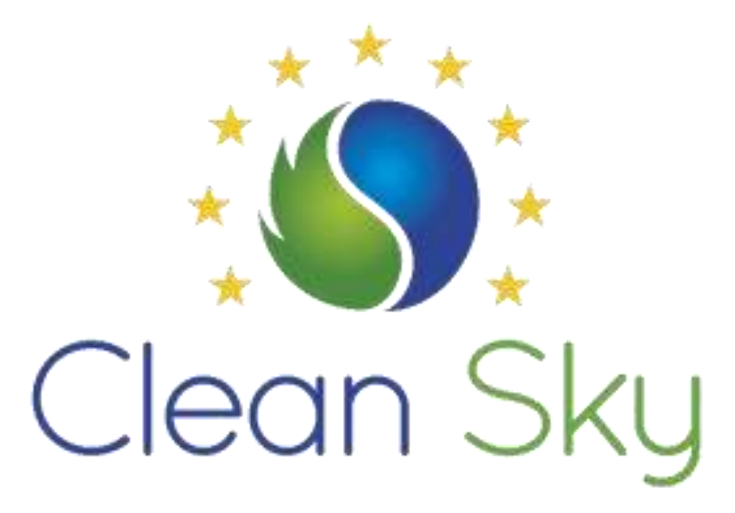

\title{
Multi-Sector Windings Bearing Relief E-Machine: Saturation and Cross Coupling Effects
}

Hanafy Mahmoud, Giorgio Valente, Michele Degano, Mauro Di Nardo, Chris Gerada and Barry James

This paper has been submitted and accepted at the IEEE international conference on Electrical Machines

(ICEM 2020)

This project has received funding from the Clean Sky 2 Joint Undertaking (JU) under grant agreement No 865364. The JU receives support from the European Union's Horizon 2020 research and innovation programme and the Clean Sky $2 \mathrm{JU}$ members other than the Union.

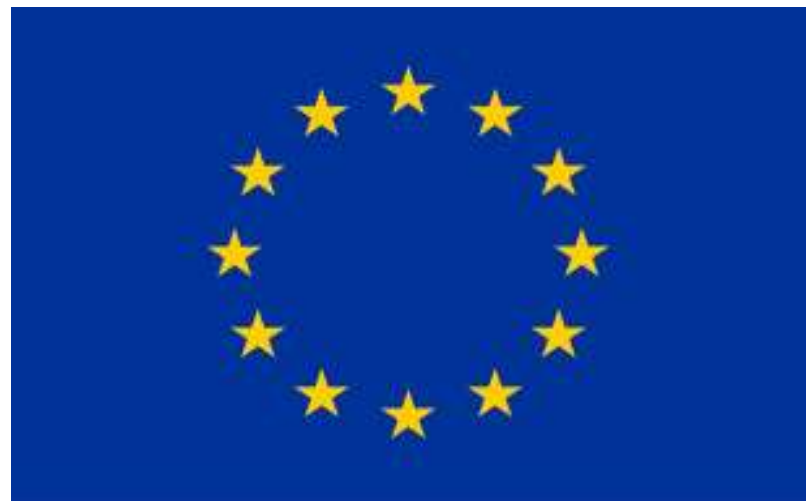




\title{
Multi-Sector Windings Bearing Relief E-Machine: Saturation and Cross Coupling Effects
}

\author{
Hanafy Mahmoud, Giorgio Valente, Michele Degano, Mauro Di Nardo, Chris Gerada and Barry James
}

\begin{abstract}
The key driving elements for any electrical machine (EM) used in aerospace applications are the reliability, mass, volume, and efficiency. Increasing the speed is a good solution for increasing the power density of the EM. Three main challenges of increasing the EM speed are: (a) the possible failure modes of the existing mechanical bearings at high speed, as well as higher friction losses, (b) the rotor displacements/whirling, which could cause toch down fault and failure of the EM, and (c) the power electronics devices switching limit.

In this paper, the multi-sector windings bearing relief electric machine (MSBRM) concept is applied to high torque density permanent magnet EM. This study deals with the highly saturated EM, which exhibits high electromagnetic nonlinearities. The main objectives of applying this MSBRM concept is to achieve the magnetic levitation on the rotor to (a) overcome the bearing reliability issues, as well as eliminating the bearing friction, (b) apply online radial force control on the rotor to mitigate the rotor displacement/whirling, and (c) reducing requirements for maintenance and monitoring.
\end{abstract}

Index Terms-Multi-Sector Windings, Bearing Relief Electric Machines, Permanent Magnet Machine, Synchronous Machine, Rotor Dynamics, Finite Element Analysis, Radial Force Control, Analytical Analyses

\section{INTRODUCTION}

The Climate Change Act of the UK government (2008) [1] [3], and the Paris Agreement (2015) [4], [5], aim to reduce UK greenhouse gas emissions by at least $80 \%$, compared to 1990 levels, by 2050. The adoption of zero emission policies set by governments is directly impacting automotive, aerospace, marine, and railway sectors. All the systems conventionally powered by combustion engines, chemical accumulators or fuel cells, will undergo drastic changes in order to meet the current and future regulations [6].

In accordance with these targets, aircraft industry id seeking for developing more fuel-efficient and environmentally friendly aircraft to alleviate pollution concerns and to comply with the goals of the 2050 strategy. The vision is to replace the traditional systems of the main generation and propulsion units, as well as the auxiliaries of the aircraft with electrical systems. In other words, the main target is to push towards more electric aircraft, both hybrid and full electric aircrafts. For both generation and propulsion systems, the electric machine requirements are very demanding in terms of power density (40kVA/kg by 2050) [7], speed, efficiency, thermal management, volume, mass, cost, and reliability.

The authors are with the Innovation and Research Department, Romax technology, Nottingham, UK. H. Mahmoud is also with the Electric Power and Machine Department, Cairo university, Giza, Egypt. Reference e-mail: Barry.James@romaxtech.com, Giorgio.Valente@romaxtech.com, and eng.hanafy4@yahoo.com.
The bearing element is one of the most critical components when dealing with high rotational speed and reliability of electrical machines. Referring to the literature review reported in [8], the magnetic levitation allows to remove the bearing mechanical friction, and reduce the monitoring and regular maintenance requirements.

The active magnetic bearings have the potential for applying the desired magnetic levitation. They are employed in several industrial and commercial applications such as compressors, flywheels, and generators, where high rotation speed is a requirement. Nevertheless, the use of the magnetic bearings generally lead to an increased overall length of the electric machine (EM), added weight, and higher cost of the drive. To this regard, multi-sector windings bearing relief electric machines (MSBRM) offer the advantage of generating both torque and radial suspension force using only a single stator structure. This reduces the volume and mass of the electric machine, and hence, maximizing the power-to-weight and power-to-volume ratio, as desired for the aerospace applications [9].

This paper studies the Bearing Relief EM (BREM) technology in the hybrid/electric aircraft. In the BREM, the rotor radial forces are controlled electromagnetically, thereby taking loads off the bearings and additionally suppressing any rotor vibrations. As a result, it is anticipated that the loads on the bearings will be lower and hence the drag produced by the bearings on the generator will be reduced, leading to an increase in generator efficiency. As a consequence, the BREM allows to run the generator at higher speeds whilst mitigating the rotor dynamics problems. This yields to use smaller air gap, which improves efficiency and thus reduces the thermal input to the motor. This is on top of the reduced bearing losses, i.e., greater efficiency and lower heat input.

Fig. 1 shows the overall control process applied to the EM in order to achieve the bearing relief operating mode. The simultaneous measurements of the rotor displacements in both $\mathrm{x}$ and $\mathrm{y}$ axes are fed to a simple PID controller, as in [9], [10]. Then, the reference values of the required forces to return the rotor back to its centre of rotation are estimated by the PID controller. These forces signals are imported to the radial force controller (RFC) controller besides to the reference operating torque. This RFC is based on the Psuedo inverse matric approach presented in [8], [9]. The outcomes of the RFC are the reference values of the independent multiple phase currents which fed to the traditional current controller of the EM.

Similar RFC algorithm has been used in [8] for low torque density surface mounted permanent magnet (PM) EM. The torque density of the studied BREM in [8]-[10] is about $20 \%$ 


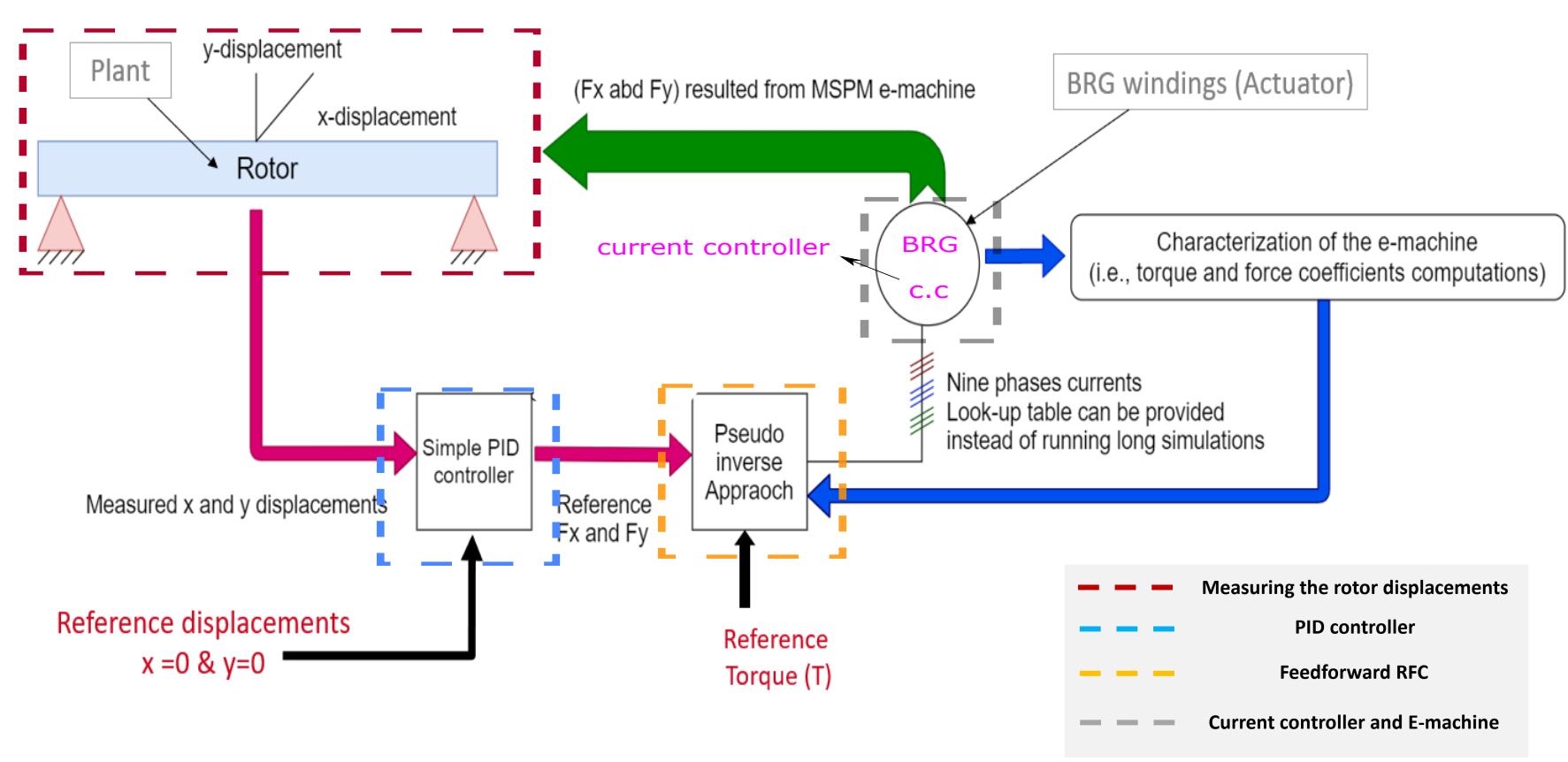

Fig. 1: Schematic diagram describes the overall control process applied to the studied MSBRM.

of the one investigated in this paper. High torque density BREM means highly saturated EM, yields to strong nonlinear magnetic characteristic.

Thus, the aim of this paper is to fill this gap with a detailed investigation of the electromagnetic characterisation of the highly saturated BREM designed for aerospace applications. In other words, it studies the bearing relief strategy for high torque density EM.

The paper is organized as follows: Section II presents the structure and the open circuit performance of the studied BREM. Section III summarises the mathematical model of the RFC algorithm using the pseudo inverse matrix approach. This section highlights the dependency of the RFC on the electromagnetic characterisation of the EM. Section IV discusses the outcomes of electromagnetic characterisation of the highly saturated BREM highlighting the magnetic nonlinearities, which were missed in the literature [8]-[10]. Section V determines the impact of the computed nonlinear electromagnetic characteristics on the accuracy of the feedforward RFC and the radial force capability of the EM. Finally, section VI concludes the analyses reported in this paper.

\section{THE STUDIED BREM STRUCTURE}

Aiming to manage the stator losses results from the high electric loading of the high torque density BERM, the stator windings are totally submerged in oil. Thus, the stator inner periphery has a retaining ring to prevent the leakage of the oil in the air-gap which would increase the windage losses. In addition, the magnetic loading of the considered BREM is increased by using Halbach array of SmCo high temperature grade. The main geometrical parameters and material data of the studied BREM are reported in Tables I and II, respectively.

From Fig. 2, it can be noted that the stator of the studied BREM has been divided into three sectors. Each sector has 12
TABLE I: Main geometrical parameters of the studied BREM.

\begin{tabular}{llll}
\hline external stator diameter & $D_{e}$ & 164 & $\mathrm{~mm}$ \\
internal stator diameter & $D_{i}$ & 96 & $\mathrm{~mm}$ \\
stack length & $L_{s t k}$ & 80.2 & $\mathrm{~mm}$ \\
number of stator slots & $Q_{s}$ & 36 & \\
number of pole pairs & $p$ & 3 & \\
air-gap length & $g$ & 2 & $\mathrm{~mm}$ \\
magnets retaining ring thickness & $t_{\text {sleeve }}$ & 4 & $\mathrm{~mm}$ \\
thickness of the PM & $t_{m}$ & 10 & $\mathrm{~mm}$ \\
SmCo remanence at $200^{\circ}$ & $B_{\text {rem }}$ & 1.14 & $\mathrm{~T}$ \\
\hline
\end{tabular}

TABLE II: Material data of the different parts of the considered BREM.

\begin{tabular}{ll}
\hline Stator lamination & JNEX900 \\
rotor shaft & $17-4 \mathrm{PH}$ \\
PM retaining ring/sleeve & Carbon fibre \\
PM grade & SmCo (Recoma 32) \\
stator windings & copper \\
stator inner surface sleeve & Carbon fibre \\
\hline
\end{tabular}

slots which accommodate three phase double layer windings, i.e., 2 slots per pole per phase. Table III reports the winding matrix of the first sector three phase winding set. Fig. 3 shows the winding connection of the first sector winding. The magnetic axis of phase a of the first sector has a mechanical offset angle from the $\mathrm{x}$-axis called $\gamma_{s}$.

The open circuit performance of the investigated EM has been evaluated by finite element analyses. The flux density distribution map is presented in Fig. 4. The air-gap flux density has also been evaluated at the middle of the air-gap length, as shown in Fig. 5.

TABLE III: First winding sector slot matrix.

\begin{tabular}{|c|c|c|c|c|c|c|c|c|c|c|c|c|}
\hline$K_{a}$ & 0 & -0.5 & -1 & -0.5 & 0 & 0 & 0 & 0.5 & 1 & 0.5 & 0 & 0 \\
\hline$K_{b}$ & 1 & 0.5 & 0 & 0 & 0 & -0.5 & -1 & -0.5 & 0 & 0 & 0 & 0.5 \\
\hline$K_{c}$ & 0 & 0 & 0 & 0.5 & 1 & 0.5 & 0 & 0 & 0 & -0.5 & -1 & -0.5 \\
\hline
\end{tabular}




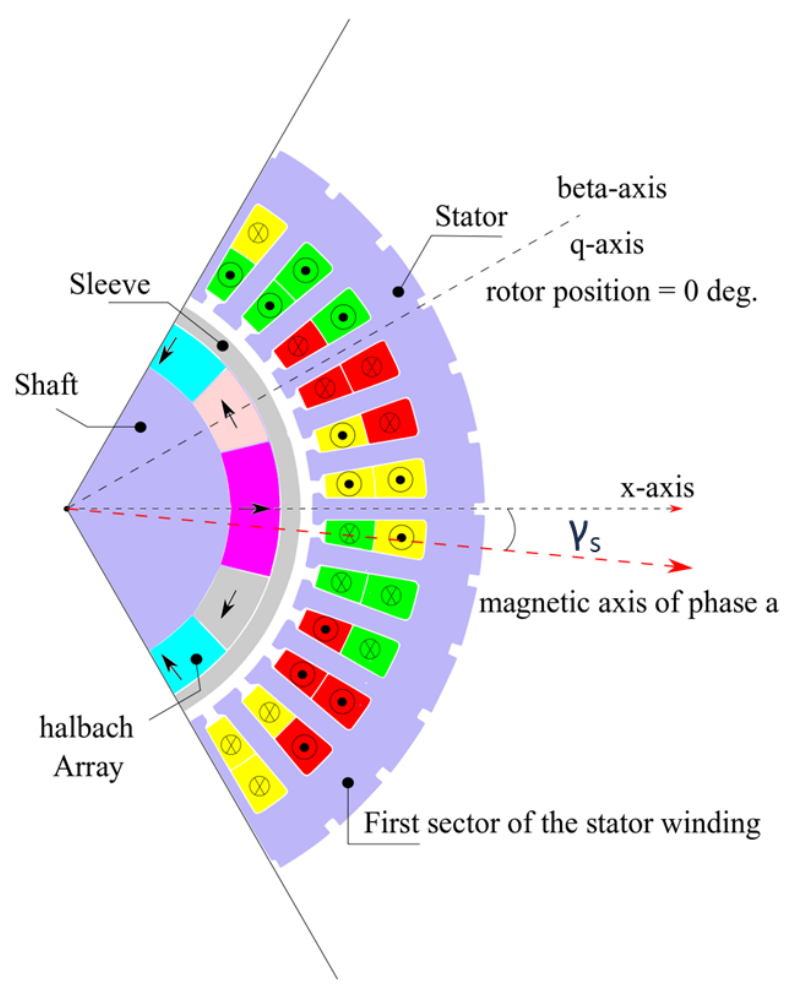

Fig. 2: 2D cross section of a single winding sector of the studied MSBRM.

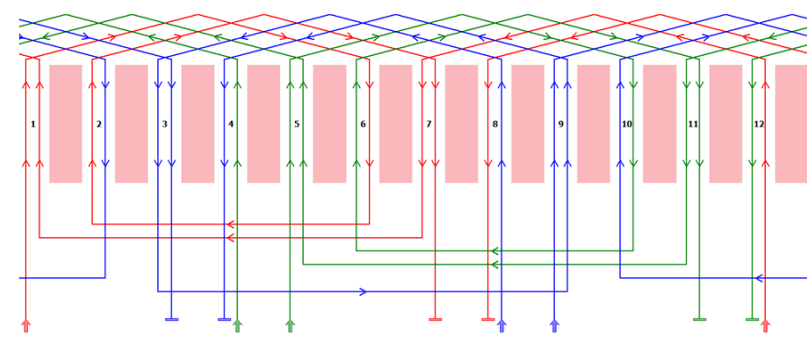

Fig. 3: Winding connection of the first sector of the MSBRM.

\section{RADIAL FORCE CONTROL}

Both Electromagnetic torque and the radial force acting on the rotor of the MSBRM are referred to as the mechanical outputs of the MSBRM. These mechanical output terms can be achieved by supplying the different winding sectors placed in the stator by independent $n s$ phase currents. As $n s$ is the total number of winding sectors. In addition, the electromagnetic characteristic of the EM itself plays an important role on the production of those mechanical outputs.

The mathematical presentation of the mechanical outputs as a function of the currents and EM characteristics has been presented in [9]. However, a linear electromagnetic characteristic of the investigated EM has been assumed in [9], as it has low torque density and saturation level. In other words, the steel laminations magnetic permeability has been set to infinity. This assumption can not be applied in the case of high torque density saturated electric machine, as the actual $\mathrm{B}-\mathrm{H}$ curve of the lamination needs to be considered in order to calculate the flux density in the different parts in the EM.

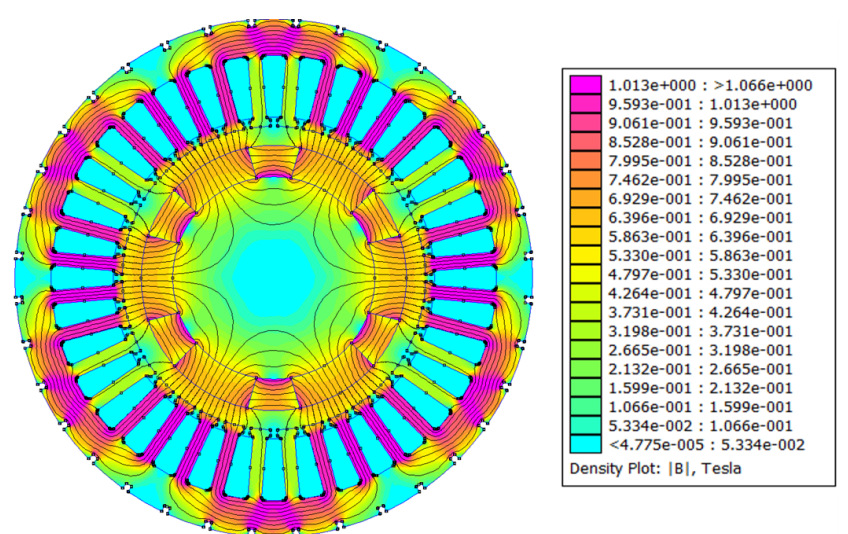

Fig. 4: Open circuit flux density distribution map of the considered MSBRM.

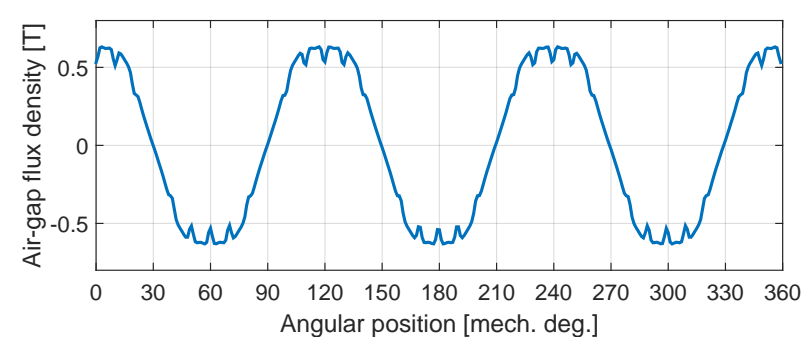

(a) Radial air-gap flux density component.

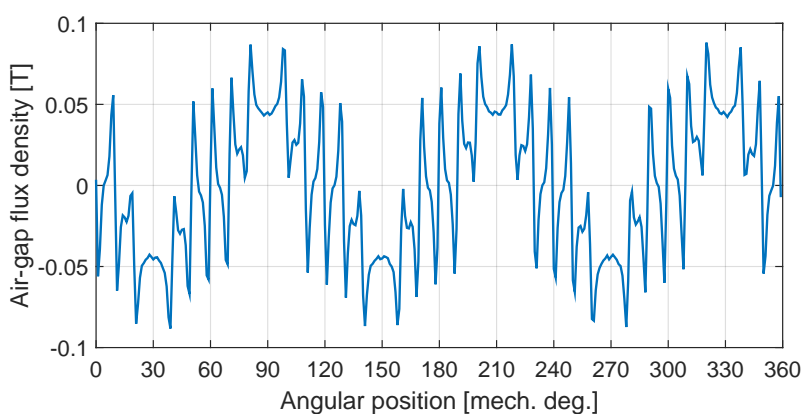

(b) Tangential air-gap flux density component.

Fig. 5: Radial and tangential flux density in the middle of the air-gap region of the analysed MSBRM.

This section summarizes the mathematical model of the RFC considering the electromagnetic nonlinearities of the studied MSBRM. In addition, it investigates the impact of these nonlinearities of the saturated MSBRM on the accuracy of the RFC.

\section{A. The Mathematical model}

The mathematical presentation of the EM mechanical outputs (radial forces and torque) can be computed as

$$
\bar{M}_{O}=\mathbf{K}_{E}\left(\vartheta_{e}, s\right) \bar{I}_{\alpha \beta}
$$

where $\vartheta_{e}$ is the electrical angular position of the rotor $\left(\vartheta_{e}=\right.$ $\left.p \vartheta_{m}\right), \bar{I}_{\alpha \beta}$ is an array that represents the stationary reference frame current components ${ }^{s} i_{\alpha}$ and ${ }^{s} i_{\beta}$ of the three stator winding sectors, and $\mathbf{K}_{E}\left(\vartheta_{e},{ }^{s} \gamma\right)$ are the electromagnetic coefficients/characteristics of the MSBRM. 
Splitting the radial force into two components in xy plane; the mechanical output array can be represented as

$$
\bar{M}_{O}=\left[\begin{array}{lll}
F_{x}\left(\vartheta_{e}\right) & F_{y}\left(\vartheta_{e}\right) & T\left(\vartheta_{e}\right)
\end{array}\right]^{T}
$$

where $F_{x}\left(\vartheta_{e}\right)$ and $F_{y}\left(\vartheta_{e}\right)$ are the forces in $\mathrm{x}$ and $\mathrm{y}$ axis, respectively.

\section{B. E-machine Electromagnetic Characterisation}

The electromagnetic torque coefficient and force coefficients of the $s^{t h}$ winding sector can be computed by feeding the current to that sector. Then, the overall torque and force coefficients of the MSBRM can be achieved by bringing the coefficients of all sectors together in a single matrix as in (3)

$$
\boldsymbol{K}_{E}=\left[{ }^{1} \boldsymbol{K}_{E}\left(\vartheta_{e}{ }^{1} \gamma\right) \ldots{ }^{n_{s}} \boldsymbol{K}_{E}\left(\vartheta_{e},{ }^{n_{s}} \gamma\right)\right]
$$

The symmetry of the three sectors of the stator windings, allows us to drive the overall coefficient matrix from the coefficients of the first sector only. This can be achieved by exploiting the rotation matrix $\mathbf{R}$ as in (4)

$$
{ }^{s} \mathbf{K}_{E}\left(\vartheta_{e},{ }^{s} \gamma\right)=\mathbf{R}\left({ }^{s} \gamma\right){ }^{1} \mathbf{K}_{E}\left(\vartheta_{e}, 0\right)
$$

As a consequence, the coefficients matrix can be rearranged as

$$
\boldsymbol{K}_{E}={ }^{1} \boldsymbol{K}_{E}\left(\vartheta_{e}{ }^{1} \gamma\right)\left[1 \cdots{ }^{n_{s}} \mathbf{R}\left({ }^{n_{s}} \gamma\right)\right]
$$

where $\mathbf{R}\left({ }^{n_{s}} \gamma\right)$ can be computed as

$$
\mathbf{R}\left({ }^{n_{s}} \gamma\right)=\left[\begin{array}{ccc}
\cos \left({ }^{n_{s}} \gamma\right) & -\sin \left({ }^{n_{s}} \gamma\right) & 0 \\
\sin \left({ }^{n_{s}} \gamma\right) & \cos \left({ }^{n_{s}} \gamma\right) & 0 \\
0 & 0 & 1
\end{array}\right]
$$

and ${ }^{1} \mathbf{K}_{E}\left(\vartheta_{e}{ }^{s} \gamma\right)$ consists of torque and forces in $\mathrm{x}$ and $\mathrm{y}$ axes and coefficients of the first winding sector, as shown below

$$
{ }^{1} \mathbf{K}_{E}\left(\vartheta_{e},{ }^{s} \gamma\right)=\left[\begin{array}{c}
{ }^{1} \mathbf{K}_{E, F}\left(\vartheta_{e},{ }^{s} \gamma\right) \\
{ }^{1} \bar{K}_{E, T}\left(\vartheta_{e}\right)
\end{array}\right]
$$

Solving (1), the reference current values can be obtained. However, inverting the rectangular matrix $\mathbf{K}_{E}$ is complex task. Thus, pseudo inverse of $\mathbf{K}_{E}$ has been applied as

$$
\mathbf{K}_{E}^{+}=\mathbf{K}_{E}^{T}\left(\mathbf{K}_{E} \mathbf{K}_{E}^{T}\right)^{-1}
$$

Finally, the reference current signals $\bar{I}_{\alpha \beta}^{*}$ can be computed as

$$
\bar{I}_{\alpha \beta}^{*}=\mathbf{K}_{E}^{+} \bar{M}_{o}^{*}=\mathbf{K}_{E}^{+} \bar{M}_{o}^{*}
$$

1) Torque coefficient of $1^{\text {st }}$ sector: The overall electromagnetic torque has been computed by varying the current amplitude, as shown in Fig. 6. In this case, all winding sectors' currents have been considered. It is noted that the three sets of the three phase windings are in phase. Thus, the overall torque can be computed similar to the normal single sector three phase machine. Consequently, the electromagnetic torque results from each winding sector can be obtained by dividing the overall torque by the number of sectors $(3$ sectors in our case), thanks to the symmetry of the three windings sectors.

Hence, the torque coefficient of the $s^{\text {th }}$ sector can be computed by dividing the electromagnetic torque of that sector by it's current. The electromagnetic torque has a linear relation versus the current, as it can be noted in Fig. 6. Consequently, the torque coefficient is varying in a narrow range, as shown in Fig. 7. In other words, the torque coefficient has been assumed as a constant value, as $0.11 \mathrm{Nm} / \mathrm{A}$ (rated condition).

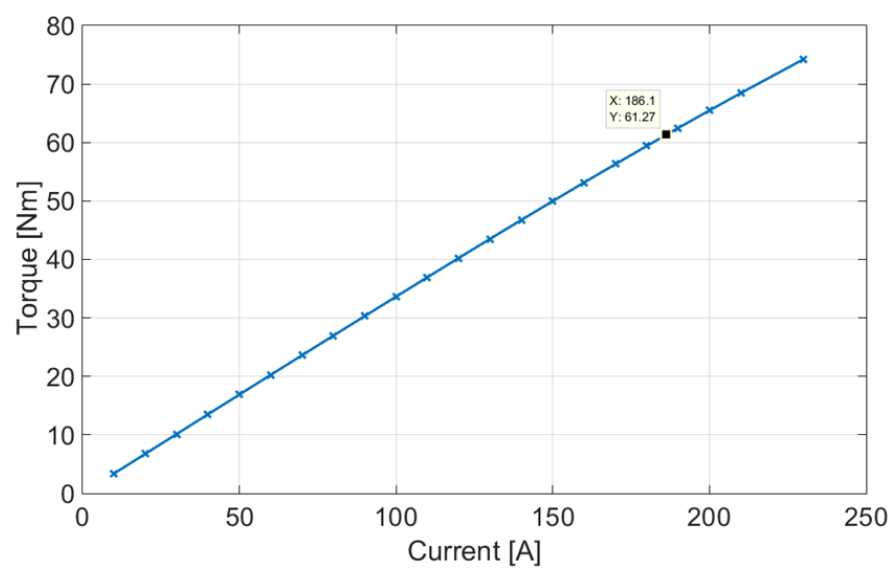

Fig. 6: Torque versus the first winding sector's current.

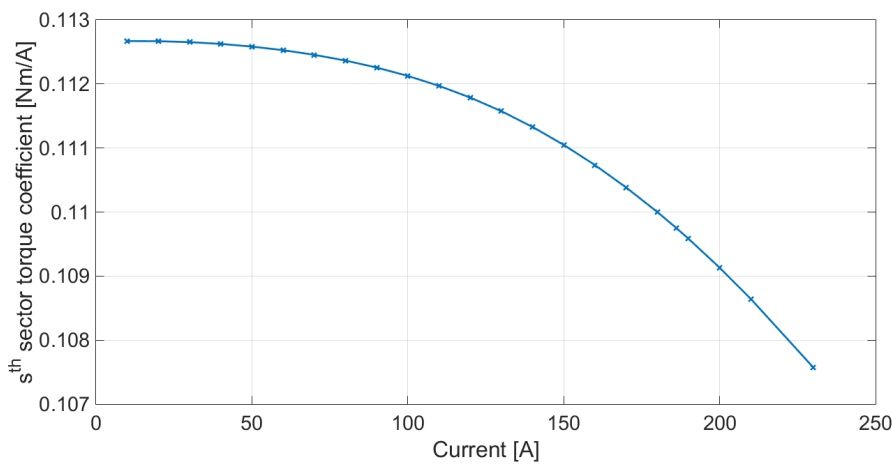

Fig. 7: First sector torque coefficient versus the current.

2) Force coefficient of $1^{\text {st }}$ sector due to $I_{\alpha}$ : Different to the torque coefficients which are based on the q-axis current only, the force coefficients in $\mathrm{x}$ and $\mathrm{y}$ axes are computed based on both $I_{\alpha}$ and $I_{\beta}$. Focusing the flux in the $\alpha$ - axis direction, both forces in x-axis $\left(F_{x \alpha}\right)$ and $\mathrm{y}$-axis $\left(F_{y \alpha}\right)$ are evaluated as shown in Fig. 8. This concentrated $\alpha$ - axis flux is generated by the $I_{\alpha}$ of the first sector windings. After that, the first sector force coefficients due to the $\alpha$ current can be computed as shown in Fig. 9. It can be noted that the coefficient $\mathbf{K}_{x \alpha}$ is highly affected by the magnetic saturation in the EM iron at high current values, whereas, the coefficient $\mathbf{K}_{y \alpha}$ is almost linear for a wide range of current values.

3) Force coefficient of $1^{\text {st }}$ sector due to $I_{\beta}$ : Analogously, the forces $F_{x \beta}$ and $F_{y \beta}$ are computed by concentrating the flux in the $\beta$-axis of the first winding sector. They are shown in Fig. 10. Once again, the magnetic saturation affects $\mathbf{K}_{x \beta}$ has a negligible impact on the $\mathbf{K}_{y \beta}$, as can be noted from Fig. 11.

\section{AnAlysis RESUlts}

The coefficients vector of the first winding sector, reported in (7), has been computed in the previous section. Then, the full coefficient matrix of the studied BREM is computed as reported in (5). Setting the desired values of the EM torque and Radial force, (9) can be solved for obtaining the independent nine phase currents of the three winding sectors. 


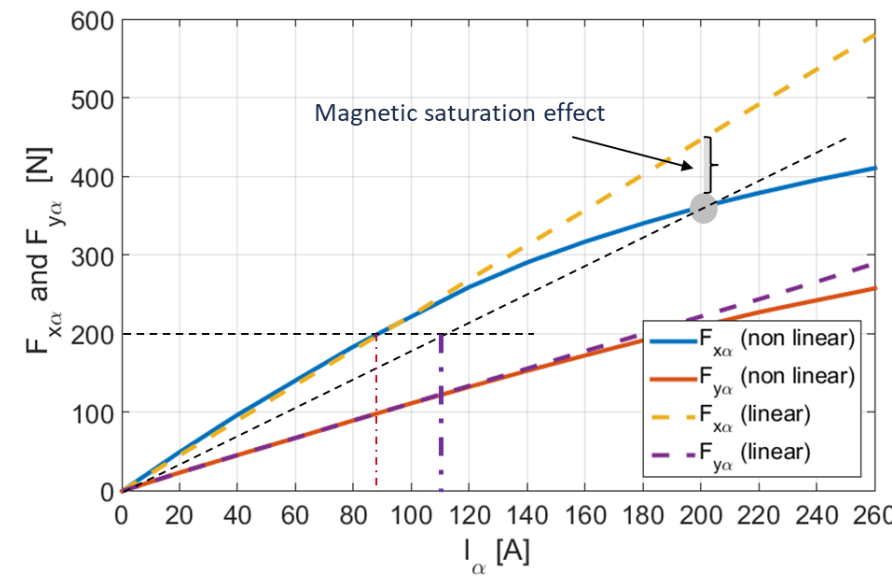

Fig. 8: Force acting on the rotor in $\alpha$-axis direction due to the first winding sector $I_{\alpha}$.

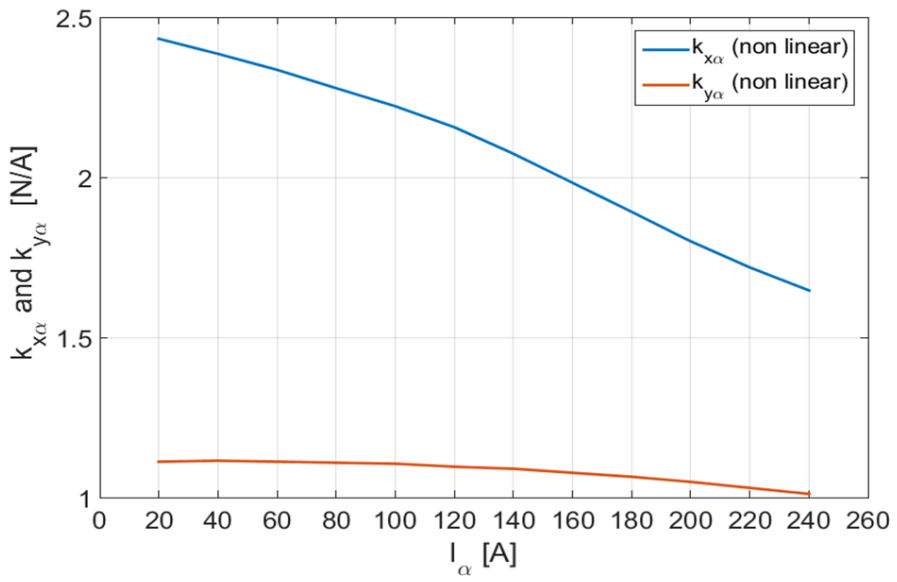

Fig. 9: Force coefficients $\mathbf{K}_{x \alpha}$ and $\mathbf{K}_{y \alpha}$ generated by $I_{\alpha}$ of the first winding sector.

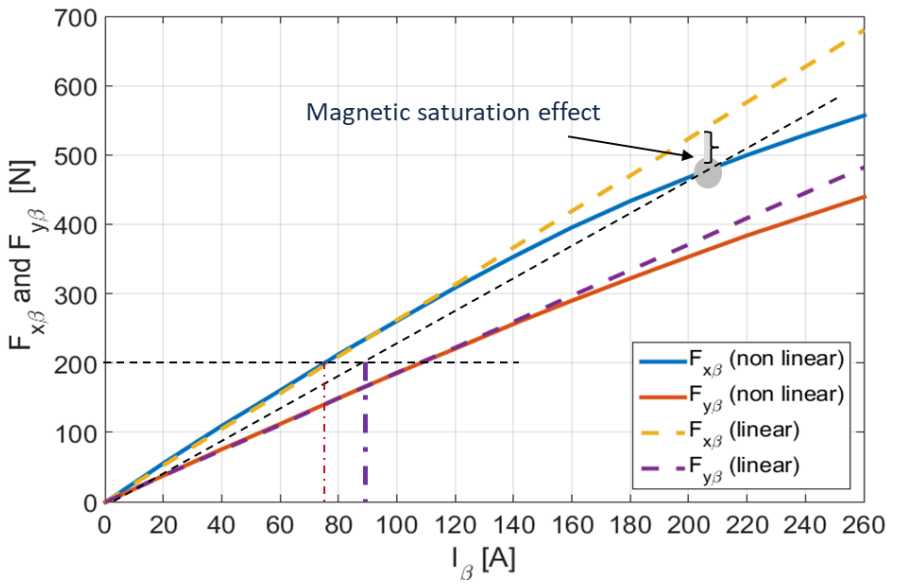

Fig. 10: Force acting on the rotor in $\beta$-axis direction due to the first winding sector $I_{\beta}$.

Different sets of the desired mechanical output of the EM has been imported to the radial force control algorithm. Then, the estimated currents for a complete electric period of the rotor rotation have been computed. These current waveforms

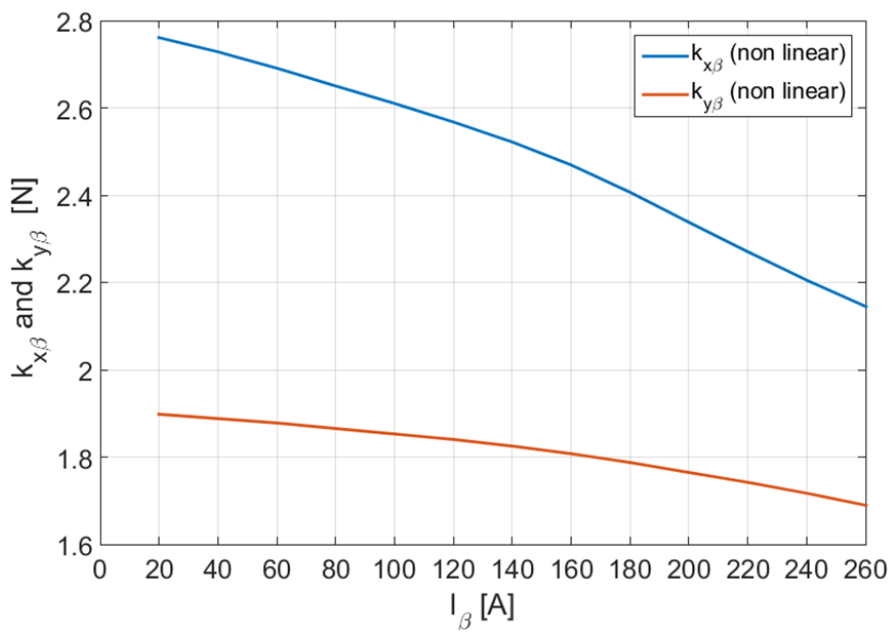

Fig. 11: Force coefficients $\mathbf{K}_{x \beta}$ and $\mathbf{K}_{y \beta}$ generated by $I_{\beta}$ of the first winding sector.

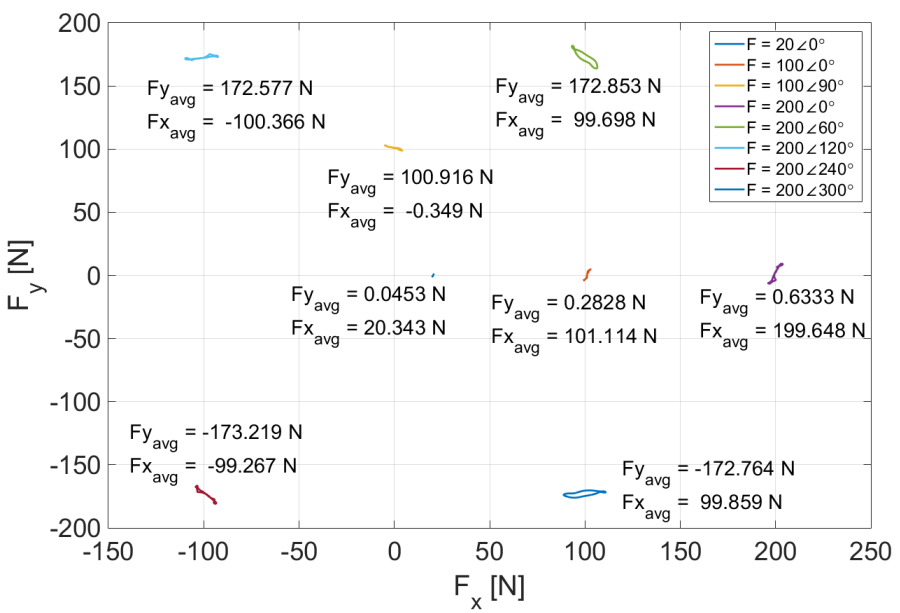

Fig. 12: Radial forces versus the rotor position.

have been imported to the finite element model of the studied EM. The evaluated radial forces by FE analysis are shown in Fig. 12. It can be note that the average value of the radial forces over one turn has a good agreement with the reference values imported to the control algorithm. In addition, it can be noted that there are oscillations in the forces waveforms around their desired values during the rotor rotation. This will be compensated simultaneously by the PID controller shown in Fig. 2 and presented in [9].

Fig. 13 and Fig. 14 show the electromagnetic decoupling between the force and torque production of the MSBRM. The radial force has not been affected by applying the torque, as in Fig. 13. Furthermore, the torque waveform has not been affected by the production of the radial force. However, the values of the torque and radial forces reported in Fig. 13 and Fig. 14 are low, i.e, the MSBRM works at the unsaturated condition reported in Fig. 8 and Fig. 10. The next two subsections discuss the nonlinearity effects on the accuracy and the decoupling between the torque and radial force production of the studied MSBRM. 


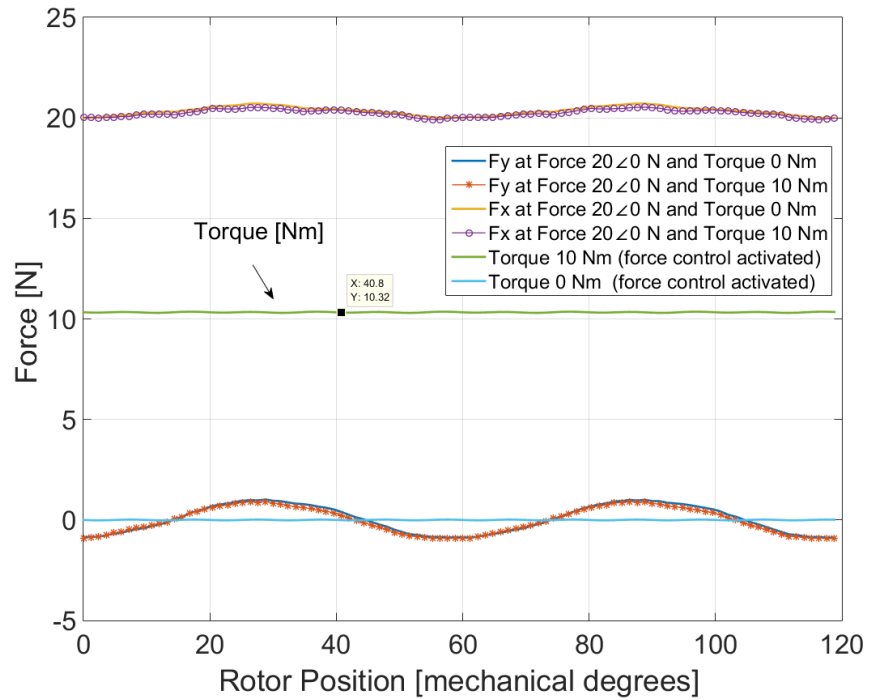

Fig. 13: Radial forces versus the rotor position with and without torque production.

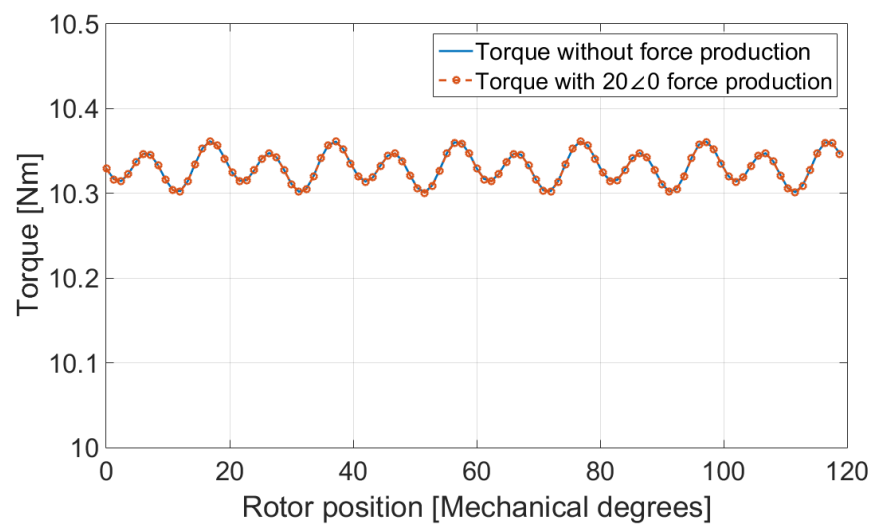

Fig. 14: Torque versus the rotor position with and without radial force production.

\section{A. Saturation Impact of the RFC}

Since the coefficients $\mathbf{K}_{x \alpha}$ and $\mathbf{K}_{x \beta}$ vary dramatically with increasing the current values, they affects the accuracy of the RFC. Therefore, this section deals with investigating the proper approach for selection these coefficients' values.

Indeed, there are two options for selecting the Coefficients $\mathbf{K}_{x \alpha}$ and $\mathbf{K}_{x \beta}$. the first option is to assign the slope of the linear part of the curve. The second one, is to consider the slope of each coefficient lies in the saturated region. Both options has been investigated setting wide range of reference radial forces.

Referring to low reference forces values, which lies in the linear part of Fig. 8 and Fig. 10, option 1 gives accurate results as shown in Fig. 13, Fig. 15, and Fig. 16. On the contrary, option 2 overestimates the radial force, as it takes into account the magnetic voltage drop of the saturated region, which does not exist in the linear part of the curve. This misleads the RFC to estimate higher current values, and hence, higher radial forces. This criteria has been highlighted in the dotted lines in Fig. 8 and Fig. 10.

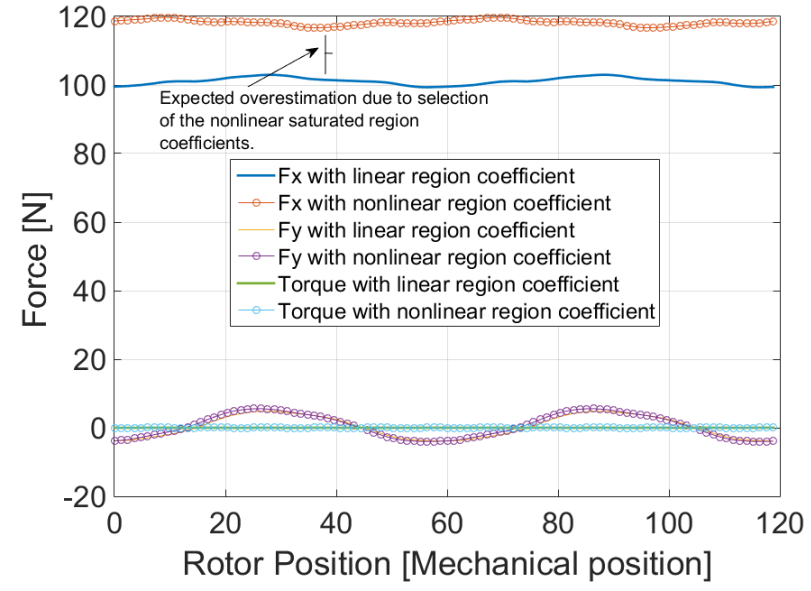

Fig. 15: Impact of the coefficients on the RFC accuracy.

Once again, referring to high reference forces values lies at the saturated part of the curve. It has been concluded that option 1 has satisfactory accuracy, as it underestimate the force in the range of $10 \%$. Besides, option 2 still overestimates the force by higher values.

This study focuses on applying radial force control not only for bearing relief, but also to compensate the unbalanced magnetic pull (UMP) results from the rotor eccentricity. The UMP has been computed at eccentricity value equal to $50 \%$ of the air-gap length. It has been concluded that, the maximum force required to compensate the UMP and the rotor weight is about $300 \mathrm{~N}$ (100N by each sector). This means most of the radial force production will lies in the linear part of the curves, considering zero torque. Next subsection discusses how the torque loading will affects accuracy of the RFC although the needed forces are within the unsaturated part.

\section{B. Cross Saturation Impact of the RFC}

Fig. 16 shows the impact of the high torque values on the radial force control. The radial force has been achieved accurately without loading the EM (with zero torque). Nevertheless, when the rated torque is produced $(60 \mathrm{Nm})$, the radial force is reduced from $200 \mathrm{Nm}$ to $180 \mathrm{~N}$ (10\% less). This is called the cross coupling saturation effect. Since the torque is set to high value, the q-axis currents of the EM will be increased significantly, as shown in Fig. 17, while, the d-axis currents of the EM don't see any significant change, as shown in Fig. 18. As a consequent, the machine becomes highly saturated and hence the magnetic voltage drop in the laminations affects the radial force production.

For a deep understand of both saturation and cross saturation effects on a wide range of torque and force production, wide series of simulations has been applied as shown in Fig. 19. It can be noted that, the saturation effect on the force accuracy is negligible for forces under $300 \mathrm{Nm}$. In addition, the cross saturation effect increases by increasing the EM torque more than $40 \mathrm{Nm}$. Furthermore, the cross coupling effect reduces the force by $10 \%$ at $60 \mathrm{Nm}$ for all level of force production, as the magnetic voltage drop results from the $200 \mathrm{~A}$ q-axis current is constant for all forces values. 


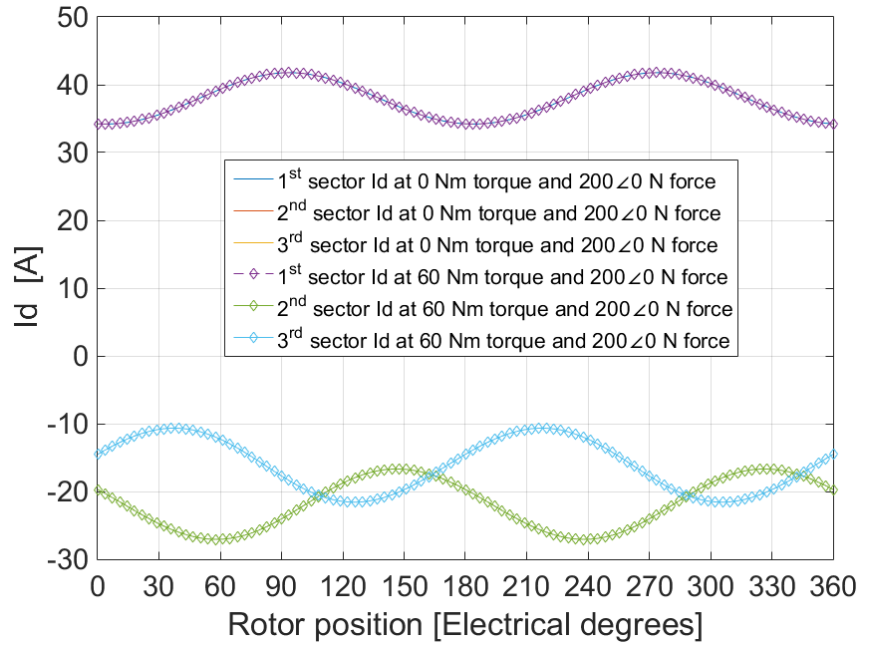

Fig. 18: The d-axis currents in case of deactivating and activating the torque production versus rotor position.

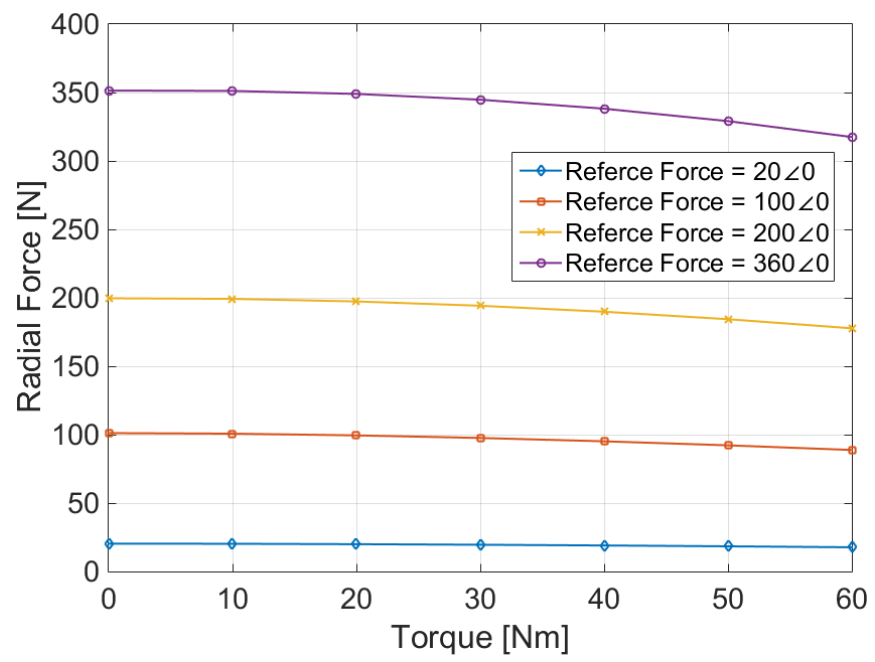

Fig. 19: Saturation and cross saturation effects on wide operating condition of the studied MSBRM.

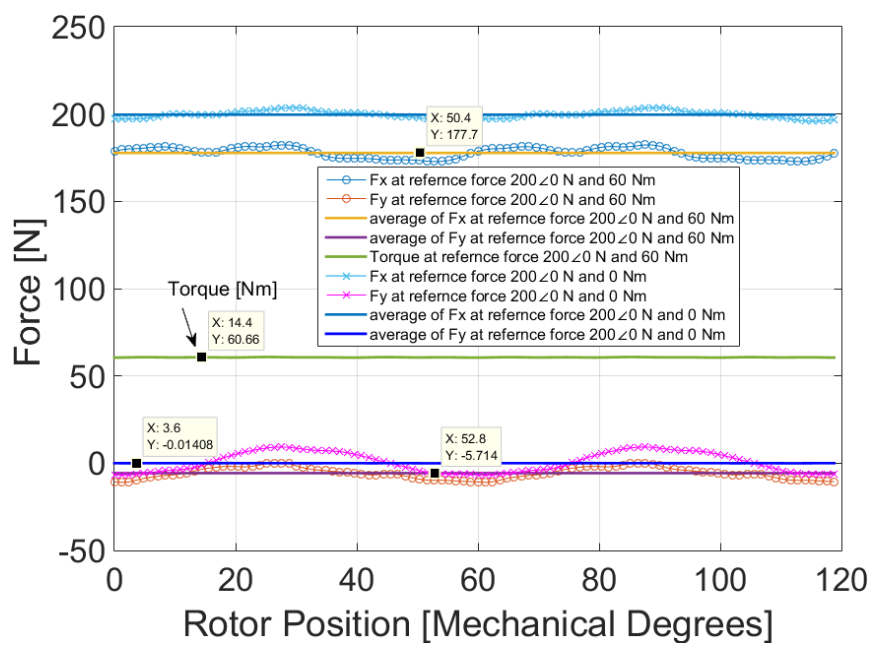

Fig. 16: Cross coupling effect between torque and radial force production.

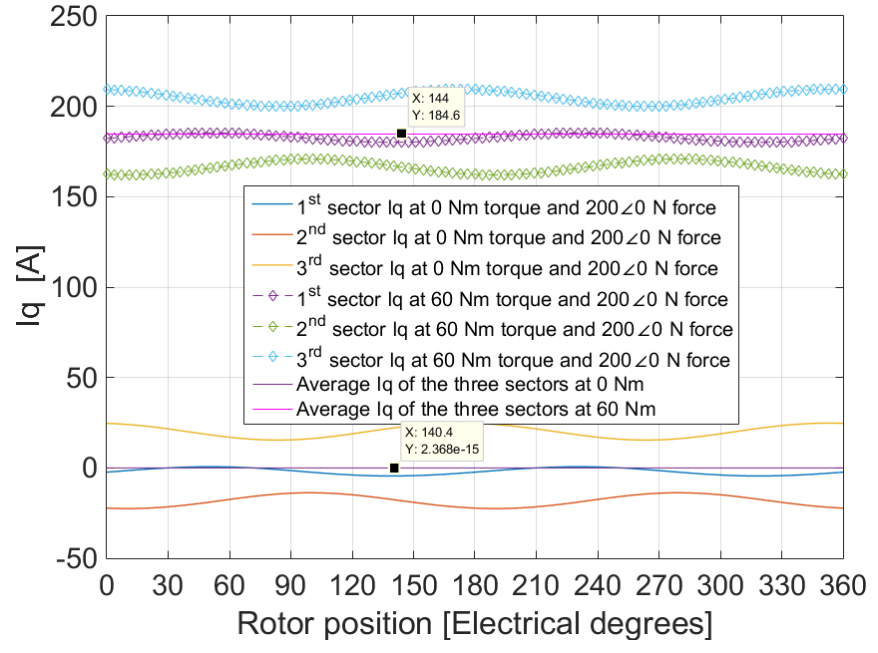

Fig. 17: The q-axis currents in case of deactivating and activating the torque production versus rotor position.

\section{CONClusion}

This paper descried the overall process of the MSBRM concept. In addition, it highlights the nonlinearities of the high torque density MSBRM and their effects on the accuracy of the radial force controller. The electromagnetic decoupling between the torque and radial force production has been approved at low electromagnetic saturation level. In addition, the electromagnetic coupling of the high saturation operating condition has been investigated. A proper selection for the electromagnetic coefficients of the highly saturated MSBRM has been recommended. Finally, a clear understand of the highly saturated EM nonlinearities on the RFC accuracy has been concluded. A wide operating range of the MSBRM has been involved. This helps to estimate their impact on the transient performance of the overall control loop and the MSBRM, as settling time, overshoot value, etc... .

\section{REFERENCES}

[1] M. Galea, C. Gerada, T. Raminosoa, and P. Wheeler, "Design of a high force density tubular permanent magnet motor," in The XIX International Conference on Electrical Machines - ICEM 2010, Sep. 2010, pp. 1-6.

[2] "http://www.legislation.gov.uk/ukpga/2008/27/contents."

[3] "https://www.gov.uk/government/publications/2010-to-2015government-policy-transport-emissions/2010-to-2015-governmentpolicy-transport-emissions."

[4] "https://en.wikipedia.org/wiki/parisagreement."

[5] N. Bianchi, E. Fornasiero, E. Carraro, S. Bolognani, and M. Castiello, "Electric vehicle traction based on a PM assisted synchronous reluctance motor," in Proceedings of IEEE International Electric Vehicle Conference (IEVC), Firenze, Italy, December 17-19, 2014, pp. 1-6.

[6] M. Hepperle, "Electric flight - potential and limitations," AVT-209 Workshop on ENERGY EFFICIENT TECHNOLOGIES AND CONCEPTS OPERATION, 2012.

[7] V. Madonna, P. Giangrande, and M. Galea, "Electrical power generation in aircraft: Review, challenges, and opportunities," IEEE Transactions on Transportation Electrification, vol. 4, no. 3, pp. 646-659, Sep. 2018.

[8] "http://eprints.nottingham.ac.uk/52086/.",

[9] G. Valente, A. Formentini, L. Papini, P. Zanchetta, and C. Gerada, "Position control study of a bearingless multi-sector permanent magnet machine," in IECON 2017 - 43rd Annual Conference of the IEEE Industrial Electronics Society, Oct 2017, pp. 8808-8813.

[10] G. Sala, G. Valente, A. Formentini, L. Papini, D. Gerada, P. Zanchetta, A. Tani, and C. Gerada, "Space vectors and pseudoinverse matrix methods for the radial force control in bearingless multisector permanent magnet machines," IEEE Transactions on Industrial Electronics, vol. 65, no. 9, pp. 6912-6922, Sep. 2018. 